\title{
ALTERAÇÕES ESTRUTURAIS E MECÂNICAS DE SOLO DE VÁRZEA CULTIVADO COM ARROZ IRRIGADO POR INUNDAÇÃO ${ }^{(1)}$
}

\author{
Marcelo Ivan Mentges ${ }^{(2)}$, José Miguel Reichert ${ }^{(3)}$, Paulo Ivonir Gubiani ${ }^{(2)}$, Dalvan José \\ Reinert $^{(3)}$ \& Alencar Xavier ${ }^{(4)}$
}

\begin{abstract}
RESUMO
A conversão de área natural para área de uso agrícola pode degradar a estrutura do solo, favorecendo a ocorrência de compactação. Este trabalho teve por objetivo avaliar as alterações nas propriedades físico-hídricas e mecânicas de um Gleissolo Háplico provocadas pela conversão de um campo natural antropizado para área de cultivo de arroz irrigado. Propriedades físico-hídricas (densidade, porosidade total, macro e microporosidade, índice de vazios e retenção de água) e mecânicas (pressão de preconsolidação, índice de compressão, coeficiente de descompressão e índice de recuperação) foram avaliadas nos horizontes $A_{1}(0,0-0,25 \mathrm{~m}), A_{2}(0,25-0,51 \mathrm{~m})$, $\mathrm{Cg}_{1}(0,51-0,92 \mathrm{~m})$ e $\mathrm{Cg}_{2}(0,92-1,20+\mathrm{m})$ em solo sob campo antropizado sem uso agrícola e nos horizontes $\mathrm{Ap}(\mathbf{0 , 0 - 0 , 1 7} \mathrm{m}), \mathrm{A}(\mathbf{0 , 1 7 - 0 , 4 0} \mathrm{m}), \mathrm{Cg}_{1}\left(\mathbf{0 , 4 0 - 0 , 7 0 ~ m ) ~ e ~} \mathrm{Cg}_{2}\right.$ $(0,70-1,00+m)$, em solo cultivado com arroz irrigado. Oito anos de cultivo de arroz sob preparo convencional e irrigação por inundação não alteraram significativamente as propriedades físico-hídricas e mecânicas, de acordo com a análise de agrupamentos. Porém, o cultivo de arroz aumentou a densidade do solo e reduziu o índice de vazios, a porosidade total e a macroporosidade. A relação da elasticidade com a densidade do solo, a porosidade total e o índice de vazios foi influenciada pelo tipo de estrutura, pois maiores compressões e descompressões ocorreram nos horizontes de estrutura prismática, em comparação aos horizontes de estrutura maciça.
\end{abstract}

Termos de indexação: pressão de preconsolidação, índice de compressão, elasticidade do solo.

(1) Parte da Dissertação de Mestrado apresentada pelo primeiro autor ao Programa de Pós-Graduação em Ciência do Solo da Universidade Federal de Santa Maria - UFSM. Financiado parcialmente pelo CNPq. Recebido para publicação em 19 de março de 2012 e aprovado em 29 de outubro de 2012.

(2) Doutorando em Ciência do Solo, UFSM. Campus Universitário. Av. Roraima, 1000, Camobi. CEP 97105-900 Santa Maria (RS). E-mail: marcelomentges@gmail.com; paulogubiani@gmail.com

(3) Professor Titular, Departamento de Solos, UFSM. E-mail: reichert@smail.ufsm.br; dalvan@ccr.ufsm.br

(4) Discente do curso de Agronomia, UFSM. E-mail: alenxav@gmail.com 


\title{
SUMMARY: STRUCTURAL AND MECHANICAL CHANGES IN A LOWLAND SOIL UNDER FLOODED RICE
}

\begin{abstract}
The conversion of natural areas for agricultural use can degrade the soil structure and increase the occurrence of compression. This study aimed to evaluate changes in the physicomechanical and hydro-physical properties of an Endoaqualf, caused by the conversion of a natural area into a rice field. Hydro-physical (density, porosity, macroporosity, microporosity, void ratio, and water retention) and mechanical properties (preconsolidation pressure, compression index, coefficient of decompression, and recovery rate) were evaluated in the horizons $A_{1}(0.0-0.25 \mathrm{~m}), A_{2}(0.25-0.51 \mathrm{~m}), C g_{1}(0.51-0.92 \mathrm{~m})$ and $C g_{2}(0.92-1.20+\mathrm{m})$ in soil of a natural area and in $\mathrm{Ap}(0.0-0.17 \mathrm{~m}), \mathrm{A}(0.17-0.40 \mathrm{~m}), \mathrm{Cg}_{1}(0.40-0.70 \mathrm{~m})$ and $C g_{2}(0.70-1.00+\mathrm{m})$ of soil under rice. Cluster analysis showed that eight years of conventionally tilled rice under flood irrigation did not significantly alter the physico-mechanical and hydro-physical properties. However, rice cultivation increased bulk density and reduced the void ratio, total porosity and macroporosity. The ratio of elasticity with bulk density, porosity and void ratio was affected by the type of soil structure, since compression and decompression were higher in horizons with prismatic than with massive structure.
\end{abstract}

Index terms: preconsolidation pressure, compression index, soil elasticity.

\section{INTRODUÇÃO}

Inúmeras propriedades físicas do solo são sensíveis ao manejo e influenciam o crescimento vegetal e as demais funções do solo no ambiente (Reichert et al., 2003). Em condições naturais, alguns solos apresentam características físicas adequadas ao desenvolvimento da vegetação nativa e de muitas espécies cultivadas (Andreola et al., 2000). No entanto, a mudança de uso e a aplicação de práticas agrícolas podem modificar significativamente a estrutura do solo, favorecendo a ocorrência de compactação (Reichert et al., 2007), diminuição dos teores de carbono (Rosa et al., 2011), estabilidade e resistência dos agregados (Nunes et al., 2002).

Solos de terras baixas, também chamadas de várzeas, em razão da drenagem deficiente, naturalmente apresentam saturação por água ou alagamentos periódicos. Essas condições são desfavoráveis para muitas culturas, porém são favoráveis ao cultivo do arroz irrigado, cultura com alta participação na produção de grãos do Estado do Rio Grande do Sul. Segundo informações do IRGA (2011), no ano agrícola 2010/2011 foram cultivados 1.117.538 ha com essa gramínea.

Grande parte das pesquisas em solos de várzea tem avaliado alterações nas propriedades físicas (Nunes et al., 2002; Pauletto et al., 2005; Lima et al., 2008, Bamberg et al., 2009). Os resultados evidenciam, de modo geral, que o uso do solo ocasiona aumento na densidade e redução na porosidade total, macroporosidade e agregação do solo. O estudo do comportamento mecânico dos solos de várzea, com alto grau de saturação, especialmente em Planossolos e Gleissolos, é incipiente; no entanto, de grande valia, uma vez que, em áreas cultivadas com arroz irrigado, o tráfego de máquinas para a aplicação de fertilizantes e defensivos e a colheita é realizado com solo saturado ou próximo da saturação.

A curva de compressão do solo permite avaliar o processo de compactação (Reichert et al., 2010) e estimar parâmetros mecânicos como a capacidade de suporte de carga, a suscetibilidade à compactação e a elasticidade, ou seja, a capacidade de recuperação da estrutura após a deformação por cargas. A avaliação de parâmetros mecânicos, juntamente com propriedades físico-hídricas, amplia o entendimento sobre o efeito do uso de solos de várzea para cultivo de arroz irrigado na estrutura do solo. Dessa forma, o objetivo deste trabalho foi avaliar as alterações nas propriedades físico-hídricas e mecânicas de um Gleissolo Háplico provocadas pela conversão de um campo antropizado para cultivo de arroz irrigado.

\section{MATERIAL E MÉTODOS}

\section{Local de estudo, delineamento experimental e tratamentos}

A área do estudo está localizada no município de Santa Maria, RS (29 43' 9'S; 53 42' 23”W; altitude média de $70 \mathrm{~m}$ ) e, segundo o Sistema Brasileiro de Classificação de Solos (Embrapa, 2006), o solo é classificado como Gleissolo Háplico. O clima da região é classificado como "Cfa" (Köppen), caracterizado por ser subtropical úmido, sem estiagem, com temperatura média do mês mais quente superior a $22^{\circ} \mathrm{C}$ e a do mês mais frio variando entre -3 e $18{ }^{\circ} \mathrm{C}$ (Moreno, 1961).

O delineamento experimental utilizado foi inteiramente casualizado. Os fatores que constituíram os tratamentos foram o uso do solo e os horizontes do 
perfil de solo. Os usos avaliados foram dois: o primeiro foi o campo antropizado sem uso agrícola (SUA), em que a vegetação predominante era composta por espécies como Eryngium sp. (gravatá), Baccharis sp. (vassoura), Erianthus sp. (macega), Melinis sp. (capim gordura), Schizachyrium condensatum (capim rabode-burro), Rosmarinus officinalis (alecrim), Aristida pallens (capim barba-de-bode), Cymbopogon sp. (capim limão), Xantium strumarium (picão), Rumex crispus (língua-de-vaca), Zanthoxylum rhoifolium (mamica de cadela), entre outras, além de espécies florestais pioneiras como a Solanum mauritianum (fumo-bravo), Myrsine lorentziana (capororoca) e Nectandra megapotamica (canela preta). Essa área caracteriza-se como campo antropizado por ter havido conversão dos fragmentos da Floresta Estacional Decidual em campo (Resolução CONAMA n ${ }^{\circ} 423$ ); contudo, não há registros no histórico da área no momento em que ocorreu a conversão. A informação disponível é que a área não é utilizada para fins agrícolas há pelo menos duas décadas. $\mathrm{O}$ segundo foi o uso agrícola para o cultivo de arroz irrigado (Oryza sativa $\mathrm{L}$.) (CAI), em que o cultivo vem sendo realizado desde 2002, no sistema convencional com inundação. $\mathrm{Na}$ primavera, o preparo do solo consiste em uma aração e quatro gradagens (até as profundidades de 0,20 e $0,10 \mathrm{~m}$, respectivamente), que são usuais para o preparo de solo nesse sistema de cultivo (SOSBAI, 2005); no inverno, a área é mantida sob pousio.

A caracterização dos perfis e as amostragens foram realizadas em maio de 2009. No campo, identificaramse os horizontes Ap (0,0-0,17 m), A (0,17-0,40 m), $\mathrm{Cg}_{1}$ $(0,40-0,70 \mathrm{~m})$ e $\mathrm{Cg}_{2}(0,70-1,00+\mathrm{m})$, para a condição CAI; e os horizontes $\mathrm{A}_{1}(0,0-0,25 \mathrm{~m}), \mathrm{A}_{2}(0,25-0,51 \mathrm{~m})$, $\mathrm{Cg}_{1}(0,51-0,92 \mathrm{~m})$ e Cg $2(0,92-1,20+\mathrm{m})$, para a condição SUA. As amostras foram extraídas do centro dos horizontes em triplicatas, totalizando 36 amostras por condição de uso do solo.

\section{Caracterização física e química}

Realizaram-se determinações de granulometria, limites de liquidez e plasticidade, índice de plasticidade e atividade da fração argila (Targ) (Embrapa, 1997) e densidade de partículas (Dp) (Gubiani et al., 2006). O teor de carbono orgânico total (C) foi determinado em um autoanalisador elementar modelo Flash 1112, enquanto a capacidade de troca de cátions em $\mathrm{pH} 7,0$ $\left(\mathrm{CTC}_{\mathrm{pH} 7}\right)$ e o teor de alumínio trocável $\left(\mathrm{Al}^{3+}\right)$ foram determinados como proposto por Tedesco et al. (1995). Os valores constam no quadro 1. Essas propriedades foram quantificadas por serem características que podem influenciar o comportamento mecânico do solo.

\section{Propriedades físico-hídricas}

A densidade (Ds), porosidade total (Pt), macroporosidade (Ma) e microporosidade (Mi) do solo foram determinadas de acordo com Embrapa (1997), em amostras com estrutura preservada, coletadas com anéis de aço inoxidável de $0,075 \mathrm{~m}$ de diâmetro e 0,04 m de altura. Nessas mesmas amostras, determinou-se o conteúdo volumétrico de água $(\theta)$ nas tensões de 1,6 e $10 \mathrm{kPa}$, em uma coluna de areia (Reinert \& Reichert, 2006), e 33 e 100 kPa, em câmara de pressão de Richards (Klute, 1986). Para as tensões de $500,1.000$ e $1.500 \mathrm{kPa}$, o conteúdo gravimétrico de água (Ug) foi determinado com potenciômetro de ponto de orvalho (dewpoint potentiometer) - WP4, em amostras de solo peneirado em malha de $2 \mathrm{~mm}$ e convertido em base volumétrica $(\theta=\mathrm{Ug} \times \mathrm{Ds})$.

A curva de retenção de água foi ajustada pelo modelo de van Genuchten (1980), cujos parâmetros empíricos $\alpha, \mathrm{n}$ e $\mathrm{m}$ do modelo foram obtidos com 0 pacote estatístico SAS (SAS Institute, 1999), considerando-se o $\theta$ na saturação e residual igual a Pt e $\theta$ na tensão de $1.500 \mathrm{kPa}$, respectivamente. A água disponível (AD) foi considerada como sendo diferença no conteúdo de água no solo entre as tensões de 10 $\mathrm{kPa}$ e $1.500 \mathrm{kPa}$, tensões essas usadas para representar, respectivamente, a capacidade de campo e o ponto de murcha permanente.

\section{Propriedades mecânicas}

O comportamento compressivo e elástico do solo foi avaliado em amostras com estrutura preservada, coletadas com anéis de aço inoxidável de $0,061 \mathrm{~m}$ de diâmetro de 0,03 $\mathrm{m}$ de altura, drenadas em coluna de areia (Reinert \& Reichert, 2006) na tensão de $10 \mathrm{kPa}$. O ensaio de compressão uniaxial foi realizado em um consolidômetro, modelo S-450 Terraload (Durham GeoInterprises). Foram aplicadas cargas sucessivas e estáticas durante $5 \mathrm{~min}$, período suficiente para atingir $99 \%$ da deformação máxima (Silva et al., 2000).

O ensaio consistiu em três etapas. Primeiramente, foi efetuado o "carregamento", com aplicação de cargas de 12,$5 ; 25 ; 50 ; 100 ; 200 ; 400$ e $800 \mathrm{kPa}$. Posteriormente, fez-se o "descarregamento" sequencial das cargas, retornando até $12,5 \mathrm{kPa}$ e, em seguida, o "recarregamento", aplicando-se as cargas de 25, 50 , $100,200,400,800$ e $1.600 \mathrm{kPa}$. Ao final do ensaio, as amostras foram secas em estufa a $105^{\circ} \mathrm{C}$ até atingirem peso constante para determinação de $\mathrm{Ds}$, índice de vazios (e) e grau de saturação (GS).

A pressão de preconsolidação $(\sigma p)$ e o índice de compressão (IC) foram determinados com as deformações da etapa do "carregamento" e da carga de $1.600 \mathrm{kPa}$ do "recarregamento". Para o conjunto de dados $(\sigma, e)$ de cada amostra, ajustou-se o modelo hidráulico de van Genuchten (1980), adaptado para as variáveis do ensaio de compressão:

$$
e=e_{f}+\left(e_{i}-\mathrm{e}_{f}\right)\left[1+(\alpha \sigma)^{n}\right]^{-m}
$$

em que $\sigma$ é a carga aplicada; $e_{i}$, o índice de vazios inicial (para $\sigma=0$ ); $e_{f}$, o índice de vazios final; e $\alpha$, n e $\mathrm{m}$, os parâmetros de ajuste. Neste estudo, aplicou-se a restrição $\mathrm{m}=1-1 / \mathrm{n}$ e $e_{f}=0$, conforme proposto por Reinert et al. (2003). Para o ajuste do modelo, foi utilizado o método não linear de Gauss-Newton, implementado no SAS (SAS Institute, 1999). 
A $\sigma p$ e o IC foram determinados de acordo com o método de Casagrande, descrito em Holtz \& Kovacs (1981), aplicado por procedimentos numéricos. A reta virgem foi definida pela reta tangente ao ponto de inflexão da curva de compressão ajustada (Gregory et al., 2006) e a $\sigma p$, estimada pelo valor de $\sigma$ na intersecção da reta virgem com a reta bissetriz. O ponto de máxima curvatura (PMC), que é a origem da bissetriz, foi definido como o maior valor da curvatura (Gregory et al., 2006):

$$
\mathrm{C}=\left|\mathrm{d}^{2} \mathrm{e} / \mathrm{d} \sigma^{2}\right| /\left[1+(\mathrm{de} / \mathrm{d} \sigma)^{2}\right]^{\frac{3}{2}}
$$

em que de/d $\sigma$ e $\mathrm{d}^{2} e / \mathrm{d}^{2} \sigma$ são a primeira e a segunda derivadas do modelo ajustado, respectivamente, sendo o PMC coincidente com o ponto de mínimo da segunda derivada da curva de compressão (Baumgartl \& Köckb, 2004). Os cálculos foram efetuados com um algoritmo desenvolvido em Visual Basic for Applications em Excel ${ }^{\circledR}$ (informações pessoais ainda não publicadas).

Os parâmetros de elasticidade do solo foram determinados com o conjunto de deformações provenientes das cargas de $800 \mathrm{kPa}$ do "carregamento", das cargas do "descarregamento" e das cargas do "recarregamento" (exceto a carga de $1.600 \mathrm{kPa}$ ). Nesse estudo, a elasticidade é expressa pelo coeficiente de descompressão $(\mathrm{Cd})$, que corresponde à declividade da reta ajustada aos dados de "descarregamento" e "recarregamento", considerando neste caso a carga de $800 \mathrm{kPa}$ como valor inicial do "descarregamento", e pelo índice de recuperação (Ir), que corresponde a razão entre a variação do índice de vazios durante o "descarregamento" e a variação do índice de vazios durante o "carregamento".

\section{Análise estatística}

O efeito do CAI na estrutura e nas variáveis físicohídricas e mecânicas do Gleissolo foi avaliado por meio da análise multivariada de agrupamentos não hierárquica. Se o CAI modifica a maioria das variáveis num mesmo sentido, o método de agrupamento das k-médias (Manly, 2008) produziria dois grupos de horizontes diferenciados pelo uso. Para essa análise, os dados foram escalonados, isto é, normalizados (escores z), em razão das diferentes escalas (unidades de medida) das variáveis. A ordem da matriz $\mathrm{X}_{(\mathrm{nxp})}$ de $\mathrm{n}$ horizontes e $\mathrm{p}$ variáveis foi $24 \mathrm{x}$ 15, quando avaliados todos os horizontes, e $12 \times 15$, para os horizontes superficiais. As 15 variáveis utilizadas foram: C, $\mathrm{Pt}, \mathrm{Ma}, \mathrm{Mi}, \mathrm{AD}$, parâmetros $\alpha$, $\mathrm{n}$ e $\mathrm{m}$ da curva de retenção de água, $\sigma \mathrm{p}, \mathrm{IC}, \mathrm{GS}, \mathrm{Ds}$, $e, \mathrm{Cd}$ e Ir, que foram representadas pelo valor médio das triplicatas de cada horizonte. Essas variáveis foram selecionadas por serem sensíveis a variações na estrutura do solo.

O efeito dos usos também foi avaliado pelo teste $t$ pareado, contrastando-se o CAI (efeito num tempo posterior) com o SUA (efeito considerado no tempo zero) no Gleissolo Háplico. Também foram feitas análises de regressão linear, introduzindo-se o tipo de estrutura como variável de classe (valor $=1$ para estrutura prismática e valor $=0$ para estrutura maciça) das variáveis mecânicas em razão das demais variáveis físico-hídricas. O número de repetições em cada análise (N) foi apresentado junto aos resultados.

\section{RESULTADOS}

\section{Alterações físico-hídricas e mecânicas}

Considerando o conjunto das variáveis físicohídricas e mecânicas avaliadas, a análise multivariada de agrupamentos não hierárquicos não agrupou os

Quadro 1. Caracterização física e química do Gleissolo Háplico nas duas condições avaliadas

\begin{tabular}{|c|c|c|c|c|c|c|c|c|c|c|c|c|}
\hline Hor. & Est. & Areia & Silte & Argila & $\mathbf{D p} \mathbf{p}^{(1)}$ & $\mathbf{L L}^{(2)}$ & $\mathbf{L} \mathbf{P}^{(\mathbf{3})}$ & $\operatorname{IP}^{(4)}$ & $\mathbf{C}^{(5)}$ & $\operatorname{Targ}^{(6)}$ & $\mathbf{A l}^{(7)}$ & $\mathrm{CTC}_{\mathrm{pH7,0}}{ }^{(8)}$ \\
\hline & & & \multirow[t]{2}{*}{$\mathrm{g} \mathrm{kg}^{-1}$} & - & $\mathrm{Mg} \mathrm{m}^{-3}$ & 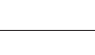 & \multirow{2}{*}{\multicolumn{2}{|c|}{$\mathrm{kg} \mathrm{kg}^{-1}$}} & \multirow[t]{2}{*}{$\%$} & \multirow{2}{*}{\multicolumn{2}{|c|}{$-\mathrm{cmol}_{\mathrm{c}} \mathrm{dm}^{-3}$}} & \\
\hline \multicolumn{7}{|c|}{ CAI } & & & & & & \\
\hline Ap & Pris. & 136 & 508 & 356 & 2,32 & 0,42 & 0,34 & 0,09 & 2,74 & 16,31 & 2,23 & 14,40 \\
\hline $\mathrm{A}$ & Pris. & 199 & 487 & 315 & 2,41 & 0,38 & 0,29 & 0,08 & 1,92 & 53,93 & 3,63 & 14,00 \\
\hline $\mathrm{Cg}_{1}$ & Mac. & 324 & 398 & 278 & 2,42 & 0,31 & 0,24 & 0,07 & 1,40 & 117,09 & 3,20 & 15,33 \\
\hline $\mathrm{Cg}_{2}$ & Mac. & 333 & 374 & 293 & 2,44 & 0,29 & 0,22 & 0,08 & 1,28 & 137,96 & 3,07 & 17,60 \\
\hline \multicolumn{13}{|c|}{ SUA } \\
\hline $\mathrm{A}_{1}$ & Pris. & 129 & 637 & 234 & 2,37 & 0,38 & 0,32 & 0,06 & 1,92 & 39,12 & 4,73 & 10,80 \\
\hline $\mathrm{A}_{2}$ & Pris. & 110 & 587 & 303 & 2,41 & 0,36 & 0,30 & 0,07 & 1,49 & 69,94 & 3,67 & 13,13 \\
\hline $\mathrm{Cg}_{1}$ & Mac. & 104 & 578 & 317 & 2,43 & 0,35 & 0,27 & 0,08 & 1,32 & 86,31 & 4,03 & 14,60 \\
\hline $\mathrm{Cg}_{2}$ & Mac. & 102 & 436 & 461 & 2,46 & 0,45 & 0,33 & 0,12 & 1,22 & 83,20 & 8,43 & 23,17 \\
\hline
\end{tabular}

Hor.: Horizonte, Est.: Estrutura, Pris.: Prismática, Mac.: Maciça, ${ }^{(1)}$ Densidade de partículas, ${ }^{(2)}$ Limite de liquidez, ${ }^{(3)}$ Limite de plasticidade, ${ }^{(4)}$ Indice de Plasticidade, ${ }^{(5)}$ Carbono orgânico total, ${ }^{(6)}$ Atividade da fração argila, ${ }^{(7)}$ Alumínio trocável e ${ }^{(8)}$ Capacidade de troca de cátions potencial. 
Quadro 2. Agrupamentos formados com horizontes de perfis de Gleissolo Háplico com e sem uso agrícola

\begin{tabular}{|c|c|}
\hline Grupo & Agrupamento $^{(1)}$ \\
\hline & Todos os horizontes \\
\hline 2 & {$\left[\underline{\mathrm{A}}, \mathrm{Cg}_{1}, \mathrm{Cg}_{\underline{1}}, \mathrm{Cg}_{\underline{1}}, \mathrm{Cg}_{\underline{2}}, \mathrm{Cg}_{\underline{2}}, \mathrm{Cg}_{\underline{2}}, \mathrm{~A}_{2}, \mathrm{Cg}_{1}, \mathrm{Cg}_{1}, \mathrm{Cg}_{1}\right]\left[\underline{\mathrm{A}}, \mathrm{A}, \mathrm{Ap}, \mathrm{Ap}, \mathrm{Ap}, \mathrm{A}_{1}, \mathrm{~A}_{1}, \mathrm{~A}_{1}, \mathrm{~A}_{2}, \mathrm{~A}_{2}, \mathrm{Cg}_{2}, \mathrm{Cg}_{2}, \mathrm{Cg}_{2}\right]$} \\
\hline 3 & {$\left[\underline{\mathrm{A}}, \mathrm{Cg}_{\underline{1}}, \mathrm{Cg}_{\underline{1}}, \mathrm{Cg}_{\underline{1}}, \mathrm{Cg}_{\underline{2}}, \mathrm{Cg}_{\underline{2}}, \mathrm{Cg}_{\underline{2}}, \mathrm{~A}_{2}, \mathrm{Cg}_{1}, \mathrm{Cg}_{1}, \mathrm{Cg}_{1}\right]\left[\underline{\mathrm{A}}, \mathrm{A}, \mathrm{Ap}, \mathrm{Ap}, \mathrm{A}_{2}, \mathrm{~A}_{2}, \mathrm{Cg}_{2}, \mathrm{Cg}_{2}, \mathrm{Cg}_{2}\right]\left[\underline{\mathrm{Ap}}, \mathrm{A}_{1}, \mathrm{~A}_{1}, \mathrm{~A}_{1}\right]$} \\
\hline \multirow[t]{2}{*}{4} & {$\left[\underline{\mathrm{Ap}}, \mathrm{A}_{1}, \mathrm{~A}_{1}, \mathrm{~A}_{1}\right]\left[\underline{\mathrm{Cg}_{1}}, \mathrm{Cg}_{\underline{1}}, \mathrm{Cg}_{\underline{1}}, \mathrm{Cg}_{\underline{2}}, \mathrm{Cg}_{\underline{2}}, \mathrm{Cg}_{\underline{2}}, \mathrm{~A}_{2}, \mathrm{Cg}_{1}, \mathrm{Cg}_{1}, \mathrm{Cg}_{1}\right]\left[\underline{\mathrm{A}}, \mathrm{A}, \mathrm{A}, \mathrm{Ap}, \mathrm{Ap}, \mathrm{A}_{2}, \mathrm{~A}_{2}, \mathrm{Cg}_{2}\right]\left[\mathrm{Cg}_{2}, \mathrm{Cg}_{2}\right]$} \\
\hline & Horizontes Ap, A, A1 e A2 \\
\hline 2 & {$\left[\underline{\mathrm{Ap}}, \mathrm{A}_{1}, \mathrm{~A}_{1}, \mathrm{~A}_{1},\right]\left[\underline{\mathrm{A}, \mathrm{A}, \mathrm{A}, \mathrm{Ap}, \mathrm{Ap}}, \mathrm{A}_{2}, \mathrm{~A}_{2}, \mathrm{~A}_{2}\right]$} \\
\hline 3 & {$[\underline{\mathrm{A}}, \mathrm{A}, \mathrm{Ap}, \mathrm{Ap}]\left[\underline{\mathrm{A}}, \mathrm{A}_{2}, \mathrm{~A}_{2}, \mathrm{~A}_{2}\right]\left[\underline{\mathrm{Ap}}, \mathrm{A}_{1}, \mathrm{~A}_{1}, \mathrm{~A}_{1}\right]$} \\
\hline 4 & {$\left[\mathrm{~A}_{1}, \mathrm{~A}_{1}, \mathrm{~A}_{1}\right]\left[\underline{\mathrm{A}}, \mathrm{A}_{2}, \mathrm{~A}_{2}, \mathrm{~A}_{2}\right][\underline{\mathrm{A}}, \underline{\mathrm{A}}, \mathrm{Ap}, \mathrm{Ap}][\underline{\mathrm{Ap}}]$} \\
\hline
\end{tabular}

(1) Os agrupamentos são formados pelos horizontes dentro dos colchetes. Horizontes com e sem sublinhado pertencem, respectivamente, aos perfis com e sem uso agrícola.

horizontes com base no uso do solo, pois os dois grupos formados reúnem horizontes com e sem uso agrícola (Quadro 2). Essa constatação é válida para o agrupamento realizado com todos os horizontes e para o agrupamento feito apenas com os horizontes superficiais. Na partição dos horizontes em três e quatro grupos, observou-se que outros fatores, possivelmente $o$ tipo de estrutura, tiveram maior efeito que o fator uso na composição dos grupos. Em um mesmo grupo houve maior similaridade dos seus elementos no que se refere ao tipo de horizonte do que ao tipo de uso.

O teste t pareado para todos os horizontes (Quadro 3 ) indicou probabilidade maior que $94 \%$ no aumento da Ds e na redução do $e$, da Pt e da Ma pelo CAI. A probabilidade de o CAI ter aumentado o GS (na tensão de $10 \mathrm{kPa}$ ) foi de $91,8 \%$. Para as demais variáveis, o teste t pareado indicou pouco efeito do uso agrícola. Para os horizontes superficiais, as probabilidades foram maiores que $96 \%$ de diminuição na $\mathrm{Ma}$, n e m e de aumento de á e maior que $92 \%$ de aumento no Ir, provocados pelo CAI.

\section{Comportamento compressivo e elástico}

$\mathrm{Na}$ avaliação do comportamento compressivo e elástico do solo, apresentaram-se somente as regressões significativas das variáveis dependentes ( $\sigma \mathrm{p}, \mathrm{IC}, \mathrm{Cd}$ e Ir) com as variáveis independentes (areia, silte, argila, carbono, Targ, $\mathrm{Ds}, \mathrm{Pt}, \mathrm{Ma}, \mathrm{Mi}, e, \mathrm{AD}, \alpha$, $\mathrm{n}, \mathrm{m}$ e GS). A regressão da $\sigma \mathrm{p}$ não foi significativa com nenhuma das variáveis independentes. Para o IC, o modelo linear foi significativo para o efeito da $\mathrm{Ds}$, da Pt, da Ma, do e e do GS (Figura 1). Para o Cd, a regressão linear foi significativa com a Pt, e, carbono, Ds, GS e Targ (Figura 2). Exceto para a Targ, as demais variáveis apresentaram efeito significativo da classe de estrutura. Apenas as variáveis GS e $\alpha$ influenciaram significativamente o $\operatorname{Ir}$ (Figura 3). $\mathrm{O}$ Ir aumentou com o decréscimo de $\alpha$ e com 0 aumento do GS.

\section{DISCUSSÃo}

A análise de agrupamento foi utilizada com o intuito de detectar se as alterações nas propriedades físico-hídricas e mecânicas, provocadas pelo cultivo de arroz irrigado, durante oito anos, foram intensas o suficiente para separar os horizontes em dois grupos distintos. Esperava-se que as arações e gradagens, aliadas ao tráfego de máquinas, teriam ocasionado alterações nas variáveis sensíveis à mudança estrutural. No entanto, os oito anos de cultivo com arroz irrigado não alteraram o padrão de similaridade dos horizontes. Os agrupamentos foram definidos muito mais pelo tipo de estrutura do que pelo efeito do uso (Quadro 2).

Quando as variáveis foram avaliadas isoladamente, houve indicação de efeito do CAI na estrutura do solo. Houve reduções no espaço poroso do solo (aumento da Ds e redução da $\mathrm{Pt}, e$ e $\mathrm{Ma}$ ) e efeitos negativos nas propriedades hídricas (parâmetros $\alpha, \mathrm{n}$ e $\mathrm{m}$ da curva de retenção) (Quadro 3). Esses efeitos se devem ao revolvimento do solo, realizado anteriormente à implantação da cultura do arroz, ao tráfego de máquinas e implementos durante a implantação, às práticas de manejo e à colheita da cultura, que influenciam a estrutura do solo. Diferentemente, na condição SUA, a atividade de organismos do solo, promotores de melhorias na agregação e porosidade desse (Reichert et al., 2007), pode ter sido fator importante para a manutenção de melhor condição estrutural do solo.

Alterações em apenas algumas propriedades físicas também foram relatadas em outras pesquisas. A conversão de campo nativo para cultivo de arroz reduziu a $\mathrm{Ma}$ e $\mathrm{Pt}$ sem causar diferenças na $\mathrm{Mi}$ em um Planossolo Háplico de textura franca (em sistema plantio direto e convencional), após 3,5 anos (Lima et al., 2008). No entanto, alterações na estrutura de Gleissolo Háplico podem não ocorrer de forma expressiva em períodos pequenos (até três anos), após a conversão de uso (Pauletto et al., 2005). Mesmo após 
Quadro 3. Médias por uso, médias das diferenças entre usos das propriedades físico-hídricas e mecânicas e significância do teste $t$ pareado para todos os horizontes e para os horizontes superficiais $\left(A p, A, A_{1}\right.$ e $\left.A_{2}\right)$ dos perfis do Gleissolo Háplico

\begin{tabular}{|c|c|c|c|c|}
\hline Variável & \multicolumn{2}{|c|}{ Média } & Média da diferença & $\mathbf{P}>\mathbf{t}$ \\
\hline \multicolumn{5}{|c|}{ Todos os horizontes } \\
\hline & SUA & CAI & & \\
\hline Densidade do solo $\left(\mathrm{Mg} \mathrm{m}^{-3}\right)$ & 1,23 & 1,33 & $-0,10$ & 0,0491 \\
\hline Porosidade total $\left(\mathrm{m}^{3} \mathrm{~m}^{-3}\right)$ & 0,511 & 0,470 & 0,041 & 0,0483 \\
\hline Macroporosidade $\left(\mathrm{m}^{3} \mathrm{~m}^{-3}\right)$ & 0,062 & 0,039 & 0,023 & 0,0561 \\
\hline Microporosidade $\left(\mathrm{m}^{3} \mathrm{~m}^{-3}\right)$ & 0,449 & 0,432 & 0,017 & 0,3321 \\
\hline Índice de vazios & 0,97 & 0,82 & 0,15 & 0,0322 \\
\hline Grau de saturação (\%) & 86,48 & 91,22 & $-4,74$ & 0,0820 \\
\hline$\alpha^{1)}$ & 0,072 & 0,062 & 0,010 & 0,6992 \\
\hline $\mathrm{n}^{(1)}$ & 1,542 & 1,547 & $-0,005$ & 0,9403 \\
\hline $\mathrm{m}^{(1)}$ & 0,346 & 0,352 & $-0,005$ & 0,8292 \\
\hline Água disponível $\left(\mathrm{m}^{3} \mathrm{~m}^{-3}\right)$ & 0,248 & 0,231 & 0,017 & 0,2412 \\
\hline Pressão de preconsolidação $(\mathrm{kPa})$ & 138,57 & 143,55 & $-4,97$ & 0,7964 \\
\hline Índice de compressão & 0,26 & 0,23 & 0,03 & 0,2466 \\
\hline Coeficiente de descompressão & 0,0340 & 0,0343 & $-0,0003$ & 0,9135 \\
\hline Índice de recuperação (\%) & 19,78 & 21,86 & $-2,07$ & 0,4128 \\
\hline \multicolumn{5}{|c|}{ Horizontes superficiais ( $A p, A, A_{1}$ e $A_{2}$ ) } \\
\hline & SUA & CAI & & \\
\hline Densidade do solo $\left(\mathrm{Mg} \mathrm{m}^{-3}\right)$ & 1,19 & 1,23 & $-0,04$ & 0,5971 \\
\hline Porosidade total $\left(\mathrm{m}^{3} \mathrm{~m}^{-3}\right)$ & 0,524 & 0,497 & 0,028 & 0,3769 \\
\hline Macroporosidade $\left(\mathrm{m}^{3} \mathrm{~m}^{-3}\right)$ & 0,085 & 0,038 & 0,048 & 0,0301 \\
\hline Microporosidade $\left(\mathrm{m}^{3} \mathrm{~m}^{-3}\right)$ & 0,439 & 0,459 & $-0,020$ & 0,2492 \\
\hline Índice de vazios & 1,02 & 0,94 & 0,08 & 0,4665 \\
\hline Grau de saturação (\%) & 80,47 & 88,41 & $-7,93$ & 0,1444 \\
\hline$\alpha^{(1)}$ & 0,107 & 0,032 & 0,075 & 0,0339 \\
\hline $\mathrm{n}^{(1)}$ & 1,462 & 1,629 & $-0,167$ & $<0,0001$ \\
\hline $\mathrm{m}^{(1)}$ & 0,315 & 0,386 & $-0,071$ & 0,0002 \\
\hline Água disponível $\left(\mathrm{m}^{3} \mathrm{~m}^{-3}\right)$ & 0,268 & 0,251 & 0,017 & 0,4325 \\
\hline Pressão de preconsolidação $(\mathrm{kPa})$ & 144,98 & 122,78 & 22,19 & 0,1932 \\
\hline Índice de compressão & 0,32 & 0,26 & 0,06 & 0,1794 \\
\hline Coeficiente de descompressão & 0,0405 & 0,0445 & $-0,0040$ & 0,5799 \\
\hline Índice de recuperação (\%) & 18,83 & 25,08 & $-6,25$ & 0,0771 \\
\hline
\end{tabular}

(1) Parâmetros empíricos da curva de retenção de água (van Genuchten, 1980). N = 24 .

sete e 19 anos de cultivo de arroz irrigado, respectivamente, em um Planossolo Solódico (Pedrotti et al., 2001) e em um Planossolo Háplico (Bamberg et al., 2009), ambos de textura média argilosa, houve aumento significativo somente na densidade na camada de 0,10-0,20 m.

A regressão linear da $\sigma p$ e do IC não foi significativa com o C, possivelmente porque a diferença na quantidade de $\mathrm{C}$ entre os usos foi pequena (Quadro 1). Porém, outros estudos demonstraram efeito significativo do material orgânico na suscetibilidade à compactação (Braida et al., 2010) e na capacidade de suporte de carga (Veiga et al., 2007).

A pequena $\mathrm{Ma}$ em ambos os usos (menor que $0,10 \mathrm{~m}^{3} \mathrm{~m}^{-3}$ ) (Quadro 3) conferiu maior número de pontos de contato entre as partículas, o que dificultou o rearranjamento e deslocamento das partículas a um estado de maior densidade (Braida et al, 2010). Embora a deformação do solo tenha relação direta com a Ma (Silva et al., 2000) e tenha havido diferença na $\mathrm{Ma}$ entre os usos (Quadro 3), o volume de Ma foi muito pequeno para que as diferenças na Ma causassem diferenças nos parâmetros de compressão.

A maior parte da Pt foi composta por Mi, que dificultou a drenagem da água e manteve elevado o GS (Quadro 3). Como o conteúdo de água do solo estava acima do limite de liquidez, o solo se comportou como um fluido no teste de compressão e a deformação dele dependeu da expulsão da água do meio poroso (Braida et al., 2010). Essa condição, que 


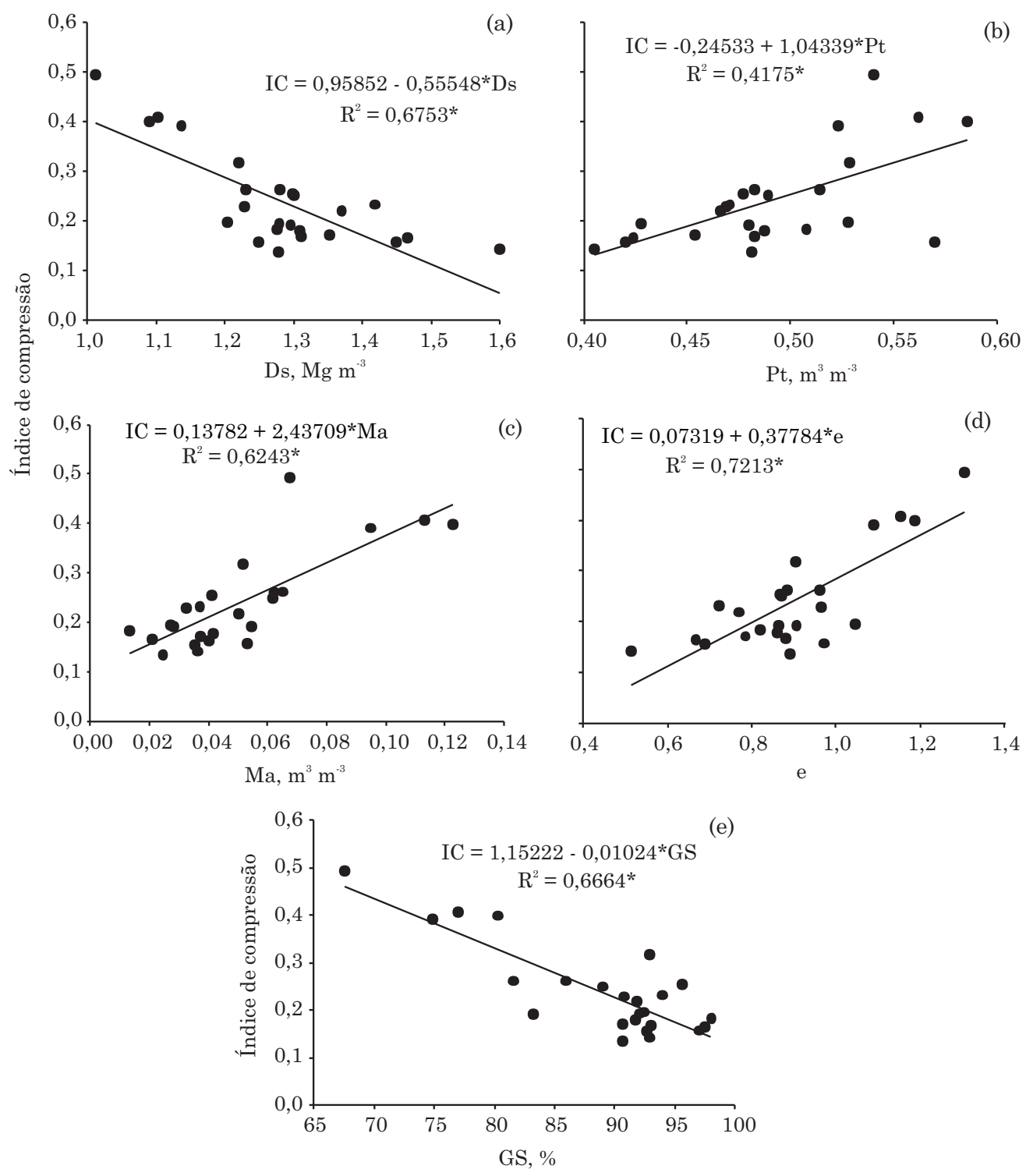

Figura 1. (a) Regressão linear do índice de compressão (IC) em razão da densidade do solo (Ds), (b) porosidade total (Pt), (c) macroporosidade (Ma), (d) índice de vazios (e) e (e) grau de saturação (GS) para o Gleissolo Háplico. ** e $^{*}$ significativo a 5 e $1 \%$, respectivamente. $\mathbf{N}=24$.

é típica de áreas cultivadas com arroz irrigado, altera o comportamento compressivo do solo em relação a solos bem drenados. Nesses, a melhor continuidade de poros e o menor grau de saturação no perfil favorecem a deformação, visto que a expulsão do ar ocorre mais facilmente que a expulsão da água para o exterior da amostra (Reichert et al., 2010). A expulsão da água do interior dos agregados durante as operações de preparo e manejo da cultura do arroz irrigado, que ocorre frequentemente em solo saturado ou próximo da saturação, pode não ter sido suficiente para que houvesse deformação e alterações expressivas nas propriedades estruturais do solo, o que explica o fato de o uso não ter influenciado no agrupamento dos horizontes (Quadro 2).
Apesar do CAI não ter alterado significativamente as propriedades mecânicas pelo teste $t$, as pequenas variações ocorridas foram suficientes para possibilitar a avaliação da relação do IC, do Cd e do Ir com as demais variáveis relacionadas com a estrutura do solo (Figuras 1, 2 e 3). Com o aumento do contato entre as partículas do solo pela compactação, houve redução da suscetibilidade à compactação, igualmente ao observado por Suzuki et al. (2008) e Saffih-Hdadi et al. (2009). Porém, esse resultado não significa melhoria estrutural e sim maior resistência a deformações quando pressões superiores a $\sigma p$ são aplicadas ao solo (Suzuki et al., 2008).

A menor suscetibilidade à compactação, quando o solo apresentou maior GS (Figura 1e), também foi 

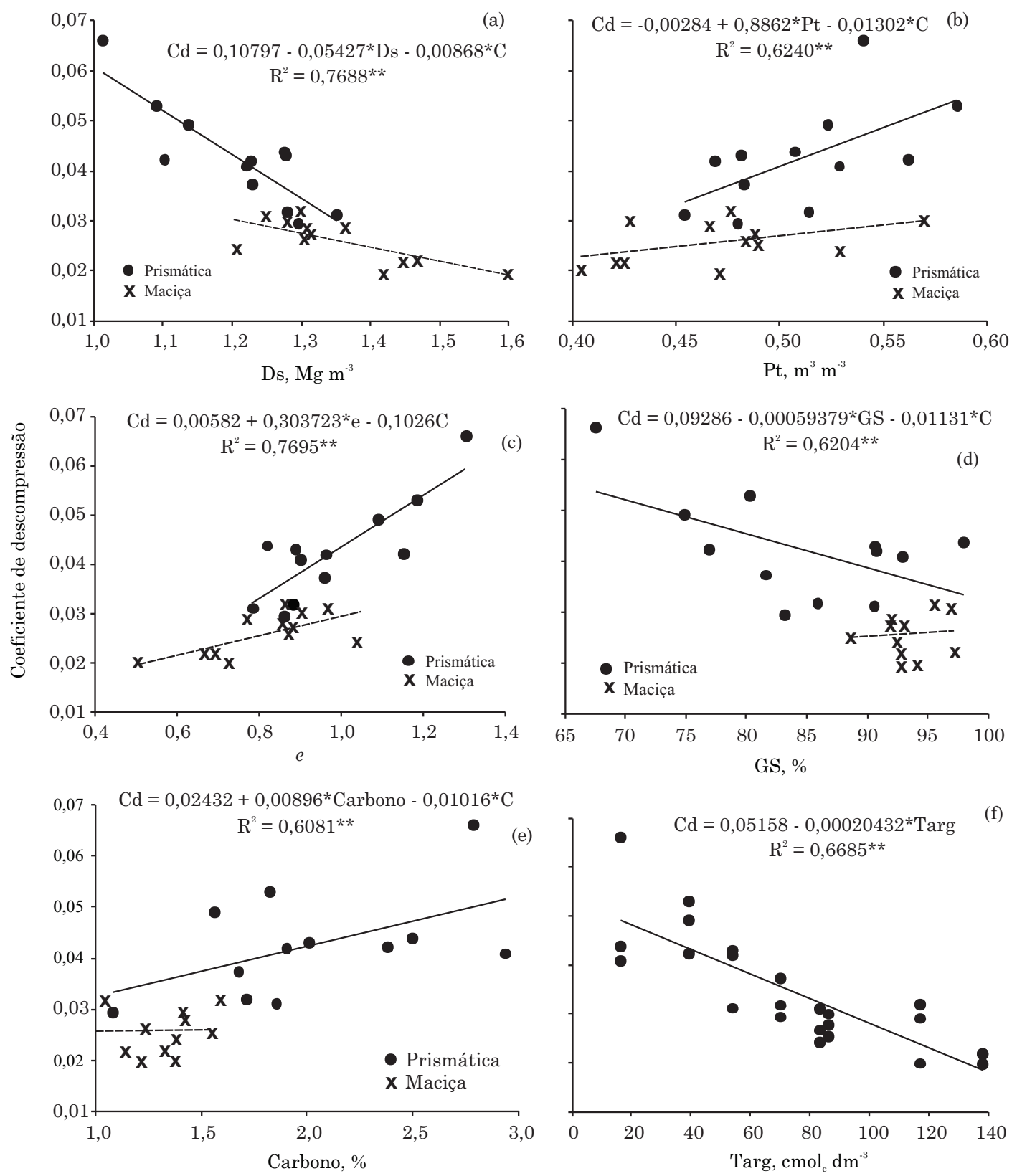

Figura 2. (a) Regressão linear do coeficiente de descompressão em razão da densidade do solo (Ds), (b) porosidade total (Pt), (c) índice de vazios (e), (d) grau de saturação (GS), (e) carbono e (f) atividade da argila (Targ) para o Gleissolo Háplico. C refere-se à classe de estrutura (valor 1,0 quando prismática e 0,0 quando maciça). ** $\mathrm{e}$ * significativo a 5 e $1 \%$, respectivamente. $\mathrm{N}=24$.

observada por Saffih-Hdadi et al. (2009). Como mencionado anteriormente, na condição de elevado grau de saturação, deformações ocorrem somente com a expulsão da água do solo, pelo fato do surgimento de pressões neutras (Braida et al., 2010). Essa constatação é importante, pois indica que a compactação do solo pode ser menos intensa quando o tráfego de máquinas em áreas cultivadas com arroz irrigado ocorre quando o solo está inundado ou próximo da saturação.

O aumento na Ds diminuiu o Cd (Figura 2a) indicando que quanto maior a quantidade de poros mais elástico é o solo, observado pela relação direta e positiva do Cd com a Pt e oe (Figura 2b,c) e negativa com o parâmetro $\alpha$ (Figura $3 \mathrm{~b})$. Porém, a relação da elasticidade com Ds, Pt e $e$ é influenciada pelo tipo de estrutura. Na estrutura maciça, a menor quantidade de poros de maior diâmetro e um contato mais íntimo entre as partículas de agregados evita que ocorram compressões e descompressões expressivas, reduzindo a elasticidade. $\mathrm{O}$ maior aprisionamento de bolhas de ar comprimido nas amostras com estrutura prismática, aumentando a elasticidade do solo, é outro efeito provável (Perdok et al., 2002). 

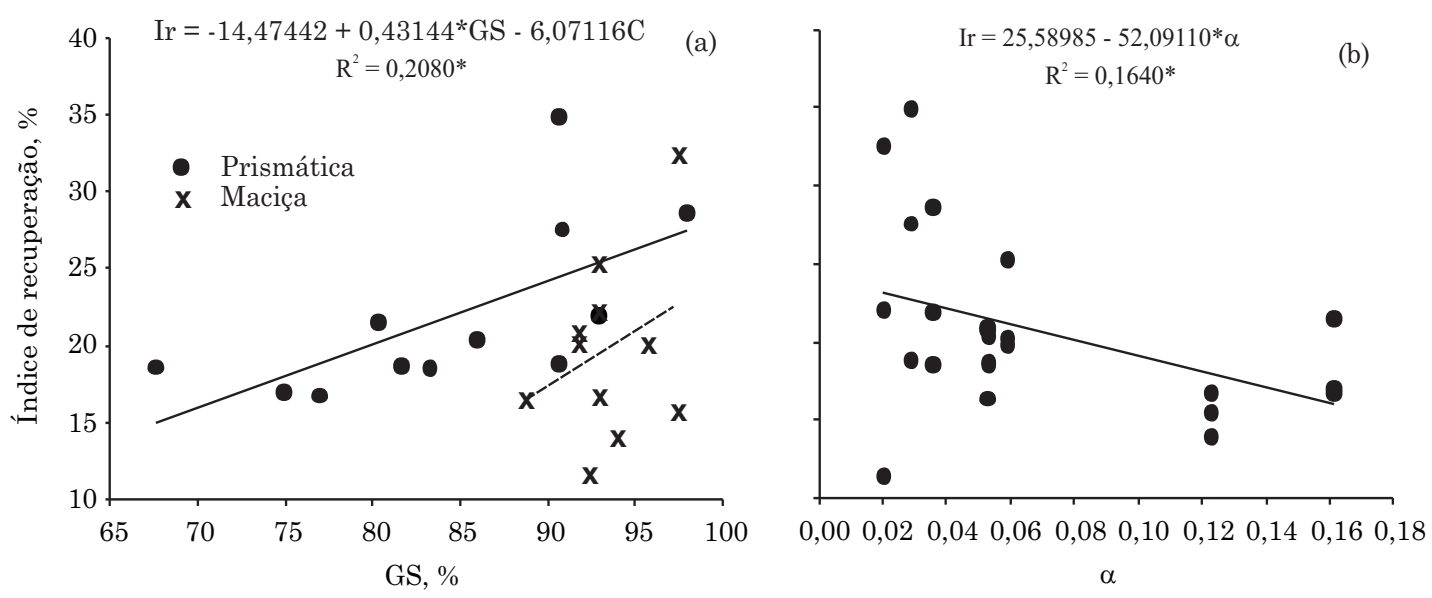

Figura 3. Regressão linear do índice de recuperação (Ir) em razão grau de saturação (GS) (a) e alpha ( $\alpha$ ) para o Gleissolo Háplico (b). C refere-se à classe de estrutura (valor 1,0 quando prismática e 0,0 quando maciça). ** e significativo a 5 e $1 \%$, respectivamente. $\mathrm{N}=\mathbf{2 4}$.

O aumento do GS aumenta o Ir (Figura 3a), mas diminui o Cd (Figura 2d), sendo essas relações dependentes do aprisionamento de bolhas de ar (Perdok et al., 2002). Porém, Braida et al. (2008) observaram aumento em ambos os indicadores de elasticidade (Ir e Cd) com o aumento do GS. A diferença no comportamento do $\mathrm{Cd}$ deste trabalho em relação ao de Braida et al. (2008) pode ser explicada pela reduzida Ma e principalmente pelo elevado GS. Neste estudo, o GS variou de aproximadamente $80 \%$ até próximo de $100 \%$, enquanto no de Braida et al. (2008); a elasticidade foi determinada em amostras praticamente secas até um GS de aproximadamente $80 \%$. A medida que aumentou o GS do Gleissolo Háplico deste estudo, as compressões e descompressões foram menores, o que ocasionou diminuição no $\mathrm{Cd}$. Quanto ao Ir, grande porcentagem de recuperação não necessariamente indicou grande elasticidade. Como as deformações foram pequenas, qualquer incremento na expansão é representativo, resultando em grandes porcentagens de recuperação. Além do GS, o tipo de estrutura também foi influente no Cd e Ir, com menor elasticidade nos horizontes de estrutura maciça, função principalmente da reduzida Ma e quantidade de C do solo.

$\mathrm{O}$ aumento no teor de $\mathrm{C}$ aumentou o $\mathrm{Cd}$ nas amostras com estrutura prismática, enquanto nas amostras com estrutura maciça, com pequena $\mathrm{Ma}$ e alto GS, o aporte de $\mathrm{C}$ não aumentou a elasticidade (Figura 2e). O material orgânico tem propriedades flexíveis e elásticas e, após o término de aplicação de uma pressão, tende a voltar à condição inicial, deslocando juntamente as partículas minerais (Soane, 1990). Além disso, segundo Braida et al. (2008), o aumento da matéria orgânica aumenta a quantidade de água retida em uma mesma tensão, facilitando o aprisionamento de bolhas de ar, aumentando a elasticidade do solo. Porém, neste trabalho, há indicações que em solos com estrutura menos desenvolvida ou com elevado estado de compactação o aporte de material orgânico pode não ser suficiente para aumentar a elasticidade.

Considerando a classificação proposta por Mitchel $\&$ Soga (2005), infere-se que nos horizontes mais profundos $\left(\mathrm{Cg}_{1}\right.$ e $\left.\mathrm{Cg}_{2}\right)$, menos influenciados pelos processos pedogenéticos, devem ocorrer argilominerais do tipo 2:1 (expansivos), em razão dos maiores valores observados de Targ (Quadro 1), enquanto próximo à superfície, onde a estrutura prismática já é definida, argilominerais como a ilita (não expansiva) devem estar presentes. Assim, esperava-se a ocorrência de maior elasticidade nos horizontes mais profundos. No entanto, comportamento contrário foi observado, ou seja, o aumento na Targ diminuiu o $\mathrm{Cd}$, indicando menor elasticidade. Portanto, a característica estrutural parece ter sido mais importante que as mineralógicas no comportamento do Cd. Dessa forma, a menor elasticidade se deve à pequena Ma e alto GS da estrutura maciça presente no Gleissolo Háplico, o que dificulta a deformação e a manifestação de maior efeito da Targ.

$O$ fato de não ter havido relação da $\sigma p$ com as variáveis avaliadas é explicada pelo alto GS e pequena $\mathrm{Ma}$, características muitas vezes observadas em solos hidromórficos. Esse comportamento é diferente do observado em solos bem drenados, onde a $\sigma p$ aumenta com a Ds e com o aumento no teor de argila (Suzuki et al., 2008) e diminui com aumento do conteúdo de água (Araújo-Junior et al., 2011) e do teor de carbono orgânico (Veiga et al., 2007).

\section{CONCLUSÕES}

1. A densidade, a porosidade, o índice de vazios e o grau de saturação foram as propriedades mais sensíveis a alterações na estrutura do Gleissolo Háplico, após oito anos de cultivo com arroz irrigado sob o sistema convencional. 
2. O índice de compressão e o coeficiente de descompressão estiveram positivamente relacionados com a porosidade e negativamente com a densidade do solo e a pressão de preconsolidação não foi afetada por nenhuma das variáveis avaliadas. $\mathrm{O}$ aumento no teor de carbono orgânico também aumentou 0 coeficiente de descompressão.

3. O tipo de estrutura deve ser considerado em estudos que envolvam parâmetros elásticos do solo, pois a elasticidade do solo foi maior no tipo de estrutura prismática em relação à maciça.

\section{AGRADECIMENTOS}

Ao CNPq, Departamento de Solos da Universidade Federal de Santa Maria e laboratório de Física do Solo dessa Instituição.

\section{LITERATURA CITADA}

ANDREOLA, F.; COSTA, L.M. \& OLSZEVSKI, N. Influência da cobertura vegetal de inverno e da adubação orgânica e, ou, mineral sobre as propriedades físicas de uma Terra Roxa Estruturada. R. Bras. Ci. Solo, 24:857-865, 2000.

ARAÚJO-JUNIOR, C.F.; DIAS JUNIOR, M.S.; GUIMARÃES, P.R.G. \& ALCÂNTARA, E.N. Capacidade de suporte de carga e umidade crítica de um Latossolo induzida por diferentes manejos. R. Bras. Ci. Solo, 35:115-131, 2011.

BAMBERG, A.L.; PAULETTO, E.A.; GOMES, A.S.; TIMM. L.C.; PINTO, L.F.S.; LIMA, A.C.R. \& SILVA, T.R. Densidade de um Planossolo sob sistemas de cultivo avaliada por meio da tomografia computadorizada de raios gama. R. Bras. Ci. Solo, 33:1079-1086, 2009.

BRAIDA, J.A.; REICHERT, J.M.; REINERT, D.J.; SEQUINATTO, L. Elasticidade do solo em função da umidade e do teor de carbono orgânico. R. Bras. Ci. Solo, $32: 477-485,2008$.

BRAIDA, J.A.; REICHERT, J.M.; REINERT, D.J. \& VEIGA, M. Teor de carbono orgânico e a susceptibilidade à compactação de um Nitossolo e um Argissolo. R. Bras. Eng. Agric. Amb., 14:131-139, 2010.

BAUMGARTL, TH. \& KÖCKB, B. Modeling Volume Change and Mechanical Properties with Hydraulic Models. Soil Sci. Soc. Am. J., 68:57-65, 2004.

EMPRESA BRASILEIRA DE PESQUISA AGROPECUÁRIA EMBRAPA. Manual de métodos de análise de solos. Rio de Janeiro, Centro Nacional de Pesquisa em Solos, 1997. 212p.

EMPRESA BRASILEIRA DE PESQUISA AGROPECUÁRIA EMBRAPA. Sistema brasileiro de classificação de solos. 2.ed. Rio de Janeiro, 2006. 306p.

GREGORY, A.S.; WHALLEY, W.R.; WATTS, C.W.; BIRD, N.R.; HALLETT, P. D. \& WHITMORE, A. Calculation of the compression index and precompression stress from soil compression test data. Soil Tillage Res., 89:45-57, 2006.
GUBIANI, P.I.; REINERT, D.J. \& REICHERT, J.M. Método alternativo para a determinação da densidade de partículas do solo - exatidão, precisão e tempo de processamento. Ci. Rural, 36:664-668, 2006.

HOLTZ, R.D. \& KOVACS, W.D. An introduction to geotechnical engineering. New Jersey: Prentice-Hall, 1981. 733p.

INSTITUTO RIO GRANDENSE DO ARROZ - IRGA. Porto Alegre, 2012. Disponível em: <http://www.irga.rs.gov.br/ uploads/anexos/1319216535Area_Produca o_ e_Produtividade.pdf> Acesso em: 8 jan. 2012.

KLUTE, A. Water retention: Laboratory methods. In: KLUTE, A., ed. Methods of soil analysis: Physical and mineralogical methods. 2.ed. Madison, American Society of Agronomy, 1986. Part. 1. p.635-662.

LIMA, C.L.R.; PILLON, C.N.; SUZUKI, L.E.A.S. \& CRUZ, L.E.C. Atributos físicos de um Planossolo Háplico sob sistemas de manejo comparados aos do campo nativo. R. Bras. Ci. Solo, 32:1849-1855, 2008.

MANLY, B.J.F. Métodos estatísticos multivariados - Uma introdução. 3.ed. Porto Alegre, Bookman, 2008. 229p.

MITCHELL, J.K. \& SOGA, K. Fundamentals of soil behavior. 3.ed. New York, John Wiley, 2005. 577p.

MORENO, J.A. Clima do Rio Grande do Sul. Porto Alegre, Secretaria da Agricultura, Diretoria de Terras e Colonizações, Secção de Geografia, 1961. 46p.

NUNES, M.L.; KLAMT, E.; REICHERT, J.M. \& DALMOLIN, R.S.D. Características de solos sistematizados em duas áreas cultivadas com arroz sob inundação. R. Bras. Ci. Solo, 26:395-406, 2002.

PAULETTO, E.A.; BORGES, J.R.; SOUSA, R.O.; PINTO, L.F.S.; SILVA, J.B. \& LEITZKE, V.W. Avaliação da densidade e da porosidade de um Gleissolo submetido a diferentes sistemas de cultivo e diferentes culturas. R. Bras. Agron., 11:207-210, 2005.

PEDROTTI, A.; PAULETTO, E.A.; GOMES, A.S.; TURATTI, A.L. \& CRESTANA, S. Sistemas de cultivo de arroz irrigado e a compactação de um Planossolo. Pesq. Agropec. Bras., 36:709-715, 2001.

PERDOK, U.D.; KROESBERGEN, B. \& HOOGMOED, W.B. Possibilities for modeling the effect of compression on mechanical and physical properties of various Dutch soil types. Soil Tillage Res., 65:61-75, 2002.

REICHERT, J.M.; REINERT, D.J.; SUZUKI, L.E.A.S. \& HORN, R. Mecânica do solo. In: van LIER, Q.J., ed. Física do solo. Viçosa, MG, Sociedade Brasileira de Ciência do Solo, 2010. p.30-102.

REICHERT, J.M.; REINERT, D.J. \& BRAIDA, J.A. Qualidade de solos e sustentabilidade de sistemas agrícolas. Ci. Amb., 27:29-48, 2003.

REICHERT, J.M.; SUZUKI, L.E.A.S. \& REINERT, D.J. Compactação do solo em sistemas agropecuários e florestais: Identificação, efeitos, limites críticos e mitigação. In: CERETTA, C.A.; SILVA, L.S. \& REICHERT, J.M., eds. Tópicos em ciência do solo. Viçosa, MG, Sociedade Brasileira de Ciência do Solo, 2007. v.5. p. 49-134, 
REINERT, D.J. \& REICHERT, J.M. Coluna de areia para medir a retenção de água no solo - protótipos e teste. Ci. Rural, 36:1931-1935, 2006.

REINERT, D.J.; ROBAINA, A.D. \& REICHERT, J.M. COMPRESS - Software e proposta de modelo para descrever a compressibilidade dos solos e seus parâmetros. In: CONGRESSO BRASILEIRO DE CIÊNCIA DO SOLO, 29., Viçosa, MG, 2003. Anais... Viçosa, MG, Sociedade Brasileira de Ciência do Solo, 2003. CD ROM.

ROSA, C.M.; CASTILHOS, R.M.V.; PAULETTO, E.A.; PILLON, C.A. \& LEAL, O.A. Conteúdo de carbono orgânico em Planossolo Háplico sob sistemas de manejo do arroz irrigado. R. Bras. Ci. Solo, 35:1769-1776, 2011.

SAFFIH-HDADI, K.; DÉFOSSEZ, P.; RICHARD, G.; CUI, Y.J.; TANG, A.M. \& CHAPLAIN, V.A. Method for predicting soil susceptibility to the compaction of surface layers as a function of water content and bulk density. Soil Tillage Res., 105:96-103, 2009.

SAS Institute. SAS/STAT procedure guide for personal computers, 5.ed. Cary, 1999. 334p.

SILVA, V.R.; REINERT, D.J. \& REICHERT, J.M. Susceptibilidade à compactação de um Latossolo Vermelho-Escuro e de um Podzólico Vermelho-Amarelo. R. Bras. Ci. Solo, 24:239-249, 2000.
SOANE, B.D. The role of organic matter in soil compactability: A review of some practical aspects. Soil Tillage Res., 16:179-201, 1990.

SOCIEDADE SUL-BRASILEIRA DE ARROZ IRRIGADO SOSBAI. Arroz irrigado: Recomendações técnicas da pesquisa para o Sul do Brasil. In: CONGRESSO BRASILEIRO DE ARROZ IRRIGADO, 4.; REUNIÃO DA CULTURA DO ARROZ IRRIGADO, 26., Santa Maria, 2005. Anais... Santa Maria, SOSBAI, 2005.

SUZUKI, L.E.A.S.; REINERT, D.J.; REICHERT, J.M. \& LIMA, C.L.R. Estimativa da susceptibilidade a compactação e do suporte de carga do solo com base em propriedades físicas de solos do Rio Grande do Sul. R. Bras. Ci. Solo, 32:963$973,2008$.

TEDESCO, M.J.; GIANELLO, C.; BISSANI, C.A.; BOHNEN, H. \& VOLKWEISS, S.J. Análise de solo, plantas e outros materiais. 2.ed. Porto Alegre, Universidade Federal do Rio Grande do Sul, 1995. 174p.

van GENUCHTEN, M.T. A closed-form equation for predicting the hydraulic conductivity of unsaturated soils. Soil Sci. Soc. Am. J., 44:892-898, 1980.

VEIGA, M.; HORN, R.; REINERT, D.J. \& REICHERT, J.M. Soil compressibility and penetrability of na Oxisol from southern Brazil, as affected by long - term tillage systems. Soil Tillage Res., 92:104-113, 2007. 